PROCEEDINGS OF THE

AMERICAN MATHEMATICAL SOCIETY

Volume 139, Number 7, July 2011, Pages 2423-2430

S 0002-9939(2010)10696-2

Article electronically published on December 20, 2010

\title{
PRESERVATION OF THE RESIDUAL CLASSES NUMBERS BY POLYNOMIALS
}

\author{
JEAN-LUC CHABERT AND YOUSSEF FARES
}

(Communicated by Matthew A. Papanikolas)

\begin{abstract}
Let $K$ be a global field and let $\mathcal{O}_{K, S}$ be the ring of $S$-integers of $K$ for some finite set $S$ of primes of $K$. We prove that whatever the infinite subset $E \subseteq \mathcal{O}_{K, S}$ and the polynomial $f(X) \in K[X]$, the subsets $E$ and $f(E)$ have the same number of residual classes modulo $\mathfrak{m}$ for almost all maximal ideals $\mathfrak{m}$ of $\mathcal{O}_{K, S}$ if and only if $\operatorname{deg}(f)=1$ when the characteristic of $K$ is 0 and $f(X)=g\left(X^{p^{k}}\right)$ for some integer $k$ and some polynomial $g$ with $\operatorname{deg}(g)=1$ when the characteristic of $K$ is $p>0$.
\end{abstract}

\section{INTRODUCTION}

In 1968, Davenport raised the following question: what can be said about $f, g \in$ $\mathbb{Z}[X]$ when, for almost all primes $p, f(\mathbb{Z}) \bmod p=g(\mathbb{Z}) \bmod p$, that is, if the set formed by the classes modulo $p$ of the elements of $f(\mathbb{Z})$ is equal to the corresponding set for $g(\mathbb{Z})$ for all but finitely many prime numbers $p$ (see [ 5 , abstract])? We are interested here in an analogous question. But first let us recall some results about Davenport's problem.

Proposition 1.1 ([3] and [] $)$. Let $f, g \in \mathbb{Z}[X]$ be such that $f(\mathbb{Z}) \bmod p=g(\mathbb{Z}) \bmod p$ for almost all primes $p$. If $f$ is not of the form $l \circ m \circ n$ where $l, m, n \in \mathbb{Z}[X]$ are of degree $\geq 2$, then $f$ and $g$ are linearly related.

Two polynomials $f$ and $g$ with coefficients in a field $L$ are said to be linearly related when there exist $a \in L^{*}$ and $b \in L$ such that $g(X)=f(a X+b)$. There are generalizations of this result:

Proposition $1.2([2])$. Let $K$ be a number field and let $\mathcal{O}_{K}$ be its ring of integers. Let $f, g \in \mathcal{O}_{K}[X]$ be such that $f\left(\mathcal{O}_{K}\right) \bmod \mathfrak{m}=g\left(\mathcal{O}_{K}\right) \bmod \mathfrak{m}$ for almost all maximal ideals $\mathfrak{m}$ of $\mathcal{O}_{K}$. If $f$ is indecomposable and if $\operatorname{deg}(f) \neq 7,11,13,15,21,31$, then $f$ and $g$ are linearly related.

Moreover, for each of these exceptional degrees, there exist counterexamples. From now on, we write f.a.a. instead of 'for almost all'. As a consequence of the previous proposition, we have:

Corollary 1.3. For every number field $K$ and every polynomial $f \in K[X]$, if $\#\left(f\left(\mathcal{O}_{K}\right) \bmod \mathfrak{m}\right)=\#\left(\mathcal{O}_{K} / \mathfrak{m}\right)$ f.a.a. $\mathfrak{m} \in \operatorname{Max}\left(\mathcal{O}_{K}\right)$, then $\operatorname{deg}(f)=1$.

Received by the editors April 11, 2010 and, in revised form, July 7, 2010.

2010 Mathematics Subject Classification. Primary 11C08; Secondary 11A07, 11R09.

Key words and phrases. Polynomial mappings, global fields, $S$-integers, $S$-units.

(C)2010 American Mathematical Society 
For a subset $E$ of $\mathcal{O}_{K}, \#(E \bmod \mathfrak{m})$ denotes the cardinality of the set $E \bmod \mathfrak{m}$ formed by the classes modulo $\mathfrak{m}$. This notation is extended to every subset $E$ of $K$ in the following way: \# $(E \bmod \mathfrak{m})$ denotes the cardinality of the set formed by the classes modulo $\mathfrak{m}\left(\mathcal{O}_{K}\right)_{\mathfrak{m}}$. Note that if the polynomial $f$ belongs to $\left(\mathcal{O}_{K}\right)_{\mathfrak{m}}[X]$, then $f\left(\mathcal{O}_{K}\right) \bmod \mathfrak{m}=\mathcal{O}_{K} \bmod \mathfrak{m}$ is equivalent to $\#\left(f\left(\mathcal{O}_{K}\right) \bmod \mathfrak{m}\right)=\#\left(\mathcal{O}_{K} \bmod \mathfrak{m}\right)$.

On the other hand, it is well known that every global field $K$ has the following property (see for instance [6, §X.11]):

Proposition 1.4. Let $K$ be a global field, let $f \in K[X]$, and let $E$ be an infinite subset of $\mathcal{O}_{K}$. If $f(E)=E$, then $\operatorname{deg}(f)=1$.

Along the lines of the last two results, we may be interested in the following generalized question:

Let $K$ be a number field, let $E$ be an infinite subset of the ring of integers $\mathcal{O}_{K}$ of $K$, and let $f \in K[X]$. Does the condition $f(E) \bmod \mathfrak{m}=E \bmod \mathfrak{m}$ for almost all maximal ideals $\mathfrak{m}$ of $\mathcal{O}_{K}$ imply that $\operatorname{deg}(f)=1$ ?

We are going to answer yes. In fact, we will consider global fields instead of number fields only, and we will use a weaker hypothesis by replacing the identity of the residue sets with the equality of their cardinalities. Note also that, in the case of a global field $K$, we will speak of primes of $K$, instead of maximal ideals of $\mathcal{O}_{K}$, in order to avoid any reference a priori to a ring of integers, especially for function fields. Our main result is the following (Theorems 3.3 and 4.4):

Theorem 1.5. Let $K$ be a global field, let $S$ be a finite set of primes of $K$, let $E$ be an infinite subset of the ring $\mathcal{O}_{K, S}$ of $S$-integers of $K$, and let $f \in K[X]$. If $f^{\prime} \not \equiv 0$, then

$$
\#(E \bmod \mathcal{P})=\#(f(E) \bmod \mathcal{P}) \text { f.a.a.primes } \mathcal{P} \text { of } K \Leftrightarrow \operatorname{deg}(f)=1 .
$$

One implication of (1) is obvious. After some preliminary remarks ( $(2)$, we will prove the reverse implication, first for number fields $(\S 3)$ and then for function fields $(\S 4)$.

Remark 1.6. On the one hand, we weaken one of the hypotheses in Proposition 1.2 by replacing the whole domain $\mathcal{O}_{K}$ with any infinite subset $E$ of $\mathcal{O}_{K}$. On the other hand, we have to assume that the degree of one of the polynomials $f$ and $g$ is one. It seems difficult to obtain an assertion with two general polynomials $f$ and $g$ because of the following example which comes from 4 . Let $f \in \mathbb{Z}[X]$ which is not injective, for instance, such that $f(0)=f(1)$. Let $h \in \mathbb{Z}[X]$ such that $h(0)=1$, for instance, $h(X)=X^{2}+X+1$. Let $g(X)=f(h(X))$ and $E=\left\{h^{n}(0) \mid n \geq 0\right\}$. Then $f(E)=g(E)$, while $f$ may be irreducible with any degree.

\section{Preliminary Remarks}

Notation. In this section $D$ denotes an integral domain with quotient field $L$. Recall that, for every subset $E$ of $L$ and every maximal ideal $\mathfrak{m}$ of $D, \#(E \bmod \mathfrak{m})$ denotes the cardinality of the set formed by the classes modulo $\mathfrak{m} D_{\mathfrak{m}}$ of the elements of $E$. For every set $\mathcal{M}$ of maximal ideals of $D$, if $E$ and $F$ are subsets of $L$,

$$
E \equiv \equiv_{\mathcal{M}} F \quad \text { will mean } \quad \#(E \bmod \mathfrak{m})=\#(F \bmod \mathfrak{m}) \text { for all } \mathfrak{m} \in \mathcal{M} .
$$

We begin with two examples. 
Examples 2.1. (a) Assume that $D$ is semi-local (and $\neq L$ ). Let $E=J(D)$ be the Jacobson radical of $D$ and let $f(X)=X^{n}$ where $n \geq 2$. Then $J(D) \equiv_{\operatorname{Max}(D)}$ $f(J(D))$, while $J(D)$ is infinite and $\operatorname{deg}(f) \geq 2$.

(b) Assume that $D$ is not of finite character, that is, that there exists a non-zero element $d \in D$ which belongs to infinitely many maximal ideals. Let $E=\mathfrak{n}$ where $\mathfrak{n}$ is any fixed maximal ideal, let $f(X)=d X$, and let $\mathcal{M}=\{\mathfrak{m} \in \operatorname{Max}(D) \mid \mathfrak{m} \neq$ $\mathfrak{n}$ and $d \in \mathfrak{m}\}$. Then $\operatorname{deg}(f)=1$, while $\mathfrak{n} \not_{\mathcal{M}} f(\mathfrak{n})$ because, for every $\mathfrak{m} \in \mathcal{M}$, $\#(\mathfrak{n} \bmod \mathfrak{m}) \geq 2$ and $\#(f(\mathfrak{n}) \bmod \mathfrak{m})=1$.

Thus, for our purpose, it is necessary to consider non-semi-local domains with finite character. The following assertions are obvious:

Lemma 2.2. Let $E$ be a subset of $D$, let $f \in L[X]$, and let $d$ be a non-zero element of $D$. If $\mathcal{M}$ is a subset of $\operatorname{Max}(D)$ such that $f \in D_{\mathfrak{m}}[X]$ for every $\mathfrak{m} \in \mathcal{M}$ and $\mathcal{N}=\{\mathfrak{m} \in \mathcal{M} \mid d \notin \mathfrak{m}\}$, then one has

$$
E \equiv_{\mathcal{M}} f(E) \Rightarrow E \equiv_{\mathcal{N}} d f(E)
$$

and

$$
E \equiv_{\mathcal{M}} f(E) \Rightarrow d E \equiv_{\mathcal{N}} g(d E)
$$

where $d E=\{d x \mid x \in E\}$ and $g(X)=f(X / d)$.

Remarks 2.3. (i) Implication (3) shows that, when $D$ is a domain with finite character, to prove that, for every $f \in K[X]$, we have the implication

$$
\#(E \bmod \mathfrak{m})=\#(f(E) \bmod \mathfrak{m}) \text { f.a.a. } \mathfrak{m} \in \operatorname{Max}(D) \Rightarrow \operatorname{deg}(f)=1,
$$

it is enough to prove this implication for every $f \in D[X]$.

(ii) Let $E \subseteq D, f \in D[X]$, and $\mathfrak{m} \in \operatorname{Max}(D)$ such that $D / \mathfrak{m}$ is finite. Clearly,

$$
\forall a, b \in D[a-b \in \mathfrak{m} \Rightarrow f(a)-f(b) \in \mathfrak{m}],
$$

and hence $f$ induces a surjective map

$$
f_{\mathfrak{m}}: E \bmod \mathfrak{m} \rightarrow f(E) \bmod \mathfrak{m},
$$

so that $\#(f(E) \bmod \mathfrak{m})=\#(E \bmod \mathfrak{m})$ means that $f_{\mathfrak{m}}$ is injective; that is,

$$
\forall a, b \in E[a-b \notin \mathfrak{m} \Rightarrow f(a)-f(b) \notin \mathfrak{m}] .
$$

Lemma 2.4. If $D$ is of finite character and $\mathcal{M}$ is infinite, then

$$
f(E) \equiv_{\mathcal{M}} E \Rightarrow f \text { is injective on } E \text {. }
$$

Proof. Let $a \neq b \in E$. Then the hypothesis on $D$ implies that there exists a maximal ideal $\mathfrak{m} \in \mathcal{M}$ such that $a-b \notin \mathfrak{m}$ and hence, by Remark 2.3 (ii), such that $f(a)-f(b) \notin \mathfrak{m}$. Consequently, $f(a) \neq f(b)$.

Lemma 2.5. Assume that the characteristic of $D$ is $p>0$. For every subset $E$ of $D$, every $\mathfrak{m} \in \operatorname{Max}(D)$, and every $f \in D[X]$, letting $g(X)=f\left(X^{p}\right)$, we have

$$
\#(E \bmod \mathfrak{m})=\#(f(E) \bmod \mathfrak{m}) \Leftrightarrow \#(E \bmod \mathfrak{m})=\#(g(E) \bmod \mathfrak{m}) .
$$

This is an obvious consequence of the fact that $a-b \in \mathfrak{m} \Leftrightarrow a^{p}-b^{p} \in \mathfrak{m}$. 
Corollary 2.6. Assume that the characteristic of $D$ is $p>0$. If, for all $f \in D[X]$ such that $f^{\prime} \not \equiv 0$, we have

$$
\#(E \bmod \mathfrak{m})=\#(f(E) \bmod \mathfrak{m}) \text { f.a.a. } \mathfrak{m} \in \operatorname{Max}(D) \Rightarrow \operatorname{deg}(f)=1,
$$

then, for all $f \in D[X]$, we have

$$
\begin{gathered}
\#(E \bmod \mathfrak{m})=\#(f(E) \bmod \mathfrak{m}) \text { f.a.a. } \mathfrak{m} \in \operatorname{Max}(D) \\
\Rightarrow f(X)=g\left(X^{p^{k}}\right) \text { for some } g \in D[X] \text { with } \operatorname{deg}(g)=1 \text { and some } k \in \mathbb{N} .
\end{gathered}
$$

Notation. For every $f \in D[X]$, let

$$
\Phi_{f}(X, Y)=\frac{f(X)-f(Y)}{X-Y} .
$$

If $\operatorname{deg}(f)=n$, we may write

$$
\Phi_{f}(X, Y)=\sum_{k=1}^{n}(X-Y)^{k-1} f_{k}(Y) \text { with } f_{k} \in D[X] \text { and } f_{1}=f^{\prime} .
$$

Proposition 2.7. Let $E \subseteq D, f \in D[X]$, and $\mathfrak{m} \in \operatorname{Max}(D)$. Assume that $\#(f(E) \bmod \mathfrak{m})=\#(E \bmod \mathfrak{m})$. Then

$$
\forall a, b \in E, a \neq b \quad\left[\Phi_{f}(a, b) \in \mathfrak{m} \Rightarrow f^{\prime}(a) \in \mathfrak{m} \text { and } f^{\prime}(b) \in \mathfrak{m}\right] .
$$

Proof. If $\Phi_{f}(a, b) \in \mathfrak{m}$, then $f(a)-f(b) \in \mathfrak{m}$. The hypothesis implies that $a-b \in \mathfrak{m}$ (see Remark 2.3 (ii)). It follows then from the equality

$$
\Phi_{f}(a, b)=f^{\prime}(b)+(a-b) f_{2}(b)+\ldots+(a-b)^{n-1} f_{n}(b)
$$

that $f^{\prime}(b) \in \mathfrak{m}$.

\section{NUMBER FIELDS}

Notation. Let $K$ be a global field, that is, a finite extension either of the rational number field $\mathbb{Q}$ or of a rational function field $\mathbb{F}_{p}(t)$ over the finite field $\mathbb{F}_{p}$.

Let $S$ be a finite set of primes of $K$ and denote by $\mathcal{O}_{K, S}$ the ring of $S$-integers of $K$, that is,

$$
\mathcal{O}_{K, S}=\bigcap_{\mathcal{P} \notin S}\left\{x \in K \mid v_{\mathcal{P}}(x) \geq 0\right\}
$$

where $\mathcal{P}$ denotes any prime of $K$ and $v_{\mathcal{P}}$ the corresponding valuation.

Obviously, the fact that two subsets $E$ and $F$ of $\mathcal{O}_{K, S}$ satisfy

$$
\#(E \bmod \mathfrak{m})=\#(F \bmod \mathfrak{m}) \text { f.a.a. } \mathfrak{m} \in \operatorname{Max}\left(\mathcal{O}_{K, S}\right)
$$

does not depend on the choice of the finite set $S$, so that we are led to introduce the notation $E \equiv_{K} F$ :

$$
E \equiv_{K} F \text { means } \#(E \bmod \mathcal{P})=\#(F \bmod \mathcal{P}) \text { f.a.a. primes } \mathcal{P} \text { of } K .
$$

Moreover, if $E \equiv_{K} F$, then, for any finite extension $L$ of $K$, we also have $E \equiv_{L} F$. Thus, we may use the following notation.

Notation. $E \equiv F$ will mean that there exists a global field $K$ and a finite set $S$ of primes of $K$ such that $E, F \subseteq \mathcal{O}_{K, S}$ and, for almost all maximal ideals $\mathfrak{m}$ of $\mathcal{O}_{K, S}$, the sets $E$ and $F$ have the same number of classes modulo $\mathfrak{m}$ (this last assertion does not depend on the choices for $K$ and $S$ ). 
Now we consider the case of number fields. In order to prove our theorem for the ring of integers of a number field, we first recall some results on $\delta$-rings [1].

Definition 3.1. An integral domain $D$ with quotient field $L$ is said to be a $\delta$-ring if, for every infinite subset $F$ of $D$, any rational function $\varphi \in L(X)$ such that $\varphi(F) \subseteq D$ admits at most one pole.

Clearly, if $D$ is a $\delta$-ring, all such rational functions which admit one pole do have the same pole $e$. Moreover, if a polynomial $g \in L[X]$ and an infinite subset $F \subseteq D$ are such that $g(F) \subseteq U(D)$ where $U(D)$ denotes the group of units of $D$, then $g$ is of the form $\lambda(X-e)^{n}$ where $\lambda, e \in L$ and $e$ is the previous unique pole.

Proposition 3.2 ([1, Corollary 18]). For every number field $K$ and every finite set $S$ of maximal ideals of $\mathcal{O}_{K}$, the ring $\mathcal{O}_{K, S}$ of $S$-integers of $K$ is a $\delta$-ring.

Note that if some rational function $\varphi \in K(X)$ such that $\varphi(F) \subseteq \mathcal{O}_{K, S}$ for some infinite subset $F$ of $\mathcal{O}_{K, S}$ admits a pole $e$, then $e$ does not depend on $\varphi$ nor on $F$.

Theorem 3.3. Let $K$ be a number field and let $S$ be a finite set of maximal ideals of $K$. Let $E$ be an infinite subset of the ring $\mathcal{O}_{K, S}$ of $S$-integers of $K$ and let $f \in K[X]$. Then,

$$
f(E) \equiv E \Rightarrow \operatorname{deg}(f)=1 .
$$

Proof. By Remark 2.3 we may assume that $f \in \mathcal{O}_{K, S}[X]$. Let $E_{1}=\{a \in E \mid$ $\left.f^{\prime}(a) \neq 0\right\}$. Since the characteristic of $K$ is $0, E_{1}$ is infinite. Fix an element $a$ in $E_{1}$ and let $T=S \cup\left\{\mathfrak{m} \in \max \left(\mathcal{O}_{K}\right) \mid f^{\prime}(a) \in \mathfrak{m} \mathcal{O}_{K, S}\right\}$. By Proposition 2.7, for every $x \in E_{1} \backslash\{a\}, \Phi_{f}(a, x) \notin \mathfrak{m}$ when $\mathfrak{m} \notin T$, that is, $\Phi_{f}(a, x) \in \mathcal{O}_{K, T}^{\times}$. Since, by Proposition 3.2. $\mathcal{O}_{K, T}$ is a $\delta$-ring, it follows from the containment $\Phi_{f}\left(a, E_{1} \backslash\{a\}\right) \subseteq$ $\mathcal{O}_{K, T}^{\times}$that the polynomial $\Phi_{f}(a, X)$ is of the form $\Phi_{f}(a, X)=\lambda(X-e)^{n-1}$ where $\lambda$ denotes the leading coefficient of $f$ and $n$ denotes its degree.

If we consider now another element $b \in E_{1}$, analogously we have $\Phi_{f}(b, X)=$ $\lambda(X-e)^{n-1}$ with the same $\lambda$ and the same $e$. Consequently, $\operatorname{deg}_{X}\left(\Phi_{f}\right)=\operatorname{deg}_{Y}\left(\Phi_{f}\right)$ $=0$ and $n=1$.

\section{FunCTION FIELDS}

Now $K$ denotes a function field with characteristic $p$ and $S$ denotes a finite set of primes of $K$. Denote by $\mathcal{O}_{K, S}$ the ring of $S$-integers of $K$. The previous proof does not work, but since the group of units $\mathcal{O}_{K, S}^{\times}$is finitely generated [7, Prop. 14.2], we may use for our proof a special case of Voloch's following result with $G=\mathcal{O}_{K, S}^{\times} \times \mathcal{O}_{K, S}^{\times}$:

Proposition 4.1 ([8, Theorem 2]). If $L$ is a field of characteristic $p>0$ finitely generated over its prime field and $G$ is a subgroup of $L^{*} \times L^{*}$ such that $\operatorname{dim}_{\mathbb{Q}} G \otimes \mathbb{Q}$ is finite, then the equation $a x+b y=1$ has at most finitely many solutions $(x, y) \in G$ unless $(a, b)^{n} \in G$ for some $n \geq 1$.

Lemma 4.2. Let $K$ be a function field and let $S$ be a finite set of primes of $K$. Let $E$ be an infinite subset of $\mathcal{O}_{K, S}$, let $f \in \mathcal{O}_{K, S}$ with degree $n \geq 2$, and let $\mathcal{M}$ be an infinite subset of $\operatorname{Max}\left(\mathcal{O}_{K, S}\right)$. If $f(E) \equiv_{\mathcal{M}} E$, then

$$
\forall \mathfrak{m} \in \mathcal{M} \forall a, b \in E\left[a-b \neq 0 \text { and } a-b \in \mathfrak{m} \Rightarrow f^{\prime}(a) \in \mathfrak{m} \text { and } f^{\prime}(b) \in \mathfrak{m}\right] .
$$


Proof. Fix $a \neq b$ in $E$. Note first that if $a-b \in \mathfrak{m}$, then $f^{\prime}(a) \in \mathfrak{m}$ is equivalent to $f^{\prime}(b) \in \mathfrak{m}$, because of the equalities

$$
\begin{aligned}
& \Phi_{f}(a, b)=f^{\prime}(a)+(b-a) f_{2}(a)+\ldots+(b-a)^{n-1} f_{n}(a), \\
& \Phi_{f}(a, b)=f^{\prime}(b)+(a-b) f_{2}(b)+\ldots+(a-b)^{n-1} f_{n}(b) .
\end{aligned}
$$

Thus, it is enough to show that $f^{\prime}(a) f^{\prime}(b) \in \mathfrak{m}$, and, to do this, we may assume that $f^{\prime}(a) f^{\prime}(b) \neq 0$.

By considering a decomposition field $L$ of $\Phi_{f}(a, X) \Phi_{f}(b, X)$, we may write

$$
\Phi_{f}(a, X)=\prod_{i=1}^{n-1}\left(X-a_{i}\right) \quad \text { and } \quad \Phi_{f}(b, X)=\prod_{j=1}^{n-1}\left(X-b_{j}\right) .
$$

Clearly, for all $i, j, \Phi_{f}\left(a, a_{i}\right)=\Phi_{f}\left(b, b_{j}\right)=0$, and hence $f\left(a_{i}\right)=f(a)$ and $f(b)=$ $f\left(b_{j}\right)$. By Lemma 2.4 $f$ is injective on $E$ and $a_{i} \neq b_{j}$ for all $i, j$.

Let $T=S \cup\left\{\mathfrak{n} \in \operatorname{Max}\left(\mathcal{O}_{K, S}\right) \mid f^{\prime}(a) f^{\prime}(b) \in \mathfrak{n}\right\}$. The set $T$ is finite because of our assumption that $f^{\prime}(a) f^{\prime}(b) \neq 0$. Then, by Proposition 2.7, for every $\mathfrak{m} \notin T$, we have: for every $x \in E \backslash\{a\}, \Phi_{f}(a, x) \notin \mathfrak{m}$; and for every $x \in E \backslash\{b\}, \Phi_{f}(b, x) \notin \mathfrak{m}$. Let $W$ be the set of primes of $L$ dividing the primes of $K$ which are in $T$. Then, for every $x \in E \backslash\{a\}$, the $x-a_{i}$ 's are $W$-units; and for every $x \in E \backslash\{b\}$, the $x-b_{j}$ 's are $W$-units, so that, for every $i, j$, the equation

$$
\frac{1}{b_{j}-a_{i}} X+\frac{1}{b_{j}-a_{i}} Y=1
$$

admits infinitely many solutions $(x, y) \in\left(\mathcal{O}_{L, W}^{\times}\right)^{2}$, namely $\left(x-a_{i}, b_{j}-x\right)$ for $x \in E \backslash\{a, b\}$. It follows from Proposition 4.1 that for all $i, j, a_{i}-b_{j} \in \mathcal{O}_{L, W}^{\times}$.

For a fixed $i$, we have

$$
\Phi_{f}\left(a_{i}, b\right)=\frac{f\left(a_{i}\right)-f(b)}{a_{i}-b}=\prod_{j=1}^{n-1}\left(a_{i}-b_{j}\right) .
$$

Consequently, $f\left(a_{i}\right)-f(b)$ is also a $W$-unit. Since $\operatorname{deg}(f) \geq 2$, there is at least one $a_{i}$, and hence $f(a)=f\left(a_{i}\right)$ implies that $f(a)-f(b)$ is a $T$-unit.

Assuming now that $a-b \in \mathfrak{m}$, we also have $f(a)-f(b) \in \mathfrak{m}$. Necessarily, $\mathfrak{m} \in T$; that is, $f^{\prime}(a) f^{\prime}(b) \in \mathfrak{m}$.

Proposition 4.3. Let $K$ be a function field, let $S$ be a finite set of primes of $K$, let $E$ be an infinite susbset of $\mathcal{O}_{K, S}$, and let $f \in K[X]$. If there exists some element $a \in E$ such that $f^{\prime}(a) \neq 0$ and such that, for infinitely many $\mathfrak{m} \in \operatorname{Max}\left(\mathcal{O}_{K, S}\right)$, there exists an element $b_{\mathfrak{m}} \in E$ with $a-b_{\mathfrak{m}} \in \mathfrak{m}$, then $\operatorname{deg}(f)=1$.

Proof. Assume that $\operatorname{deg}(f) \geq 2$. Then it follows from the previous lemma that $f^{\prime}(a) \in \mathfrak{m}$ for infinitely many $\mathfrak{m} \in \operatorname{Max}\left(\mathcal{O}_{K, S}\right)$. Thus, $f^{\prime}(a)=0$. This is a contradiction.

Theorem 4.4. Let $K$ be a function field with characteristic $p>0$ and let $S$ be a finite set of primes of $K$. For every infinite subset $E$ of $\mathcal{O}_{K, S}$ and every $f \in K[X]$, one has

$$
f(E) \equiv_{K} E \Rightarrow f(X)=g\left(X^{p^{k}}\right) \text { where } k \in \mathbb{N} \text { and } \operatorname{deg}(g)=1 .
$$


Proof. Let $D=\mathcal{O}_{K, S}$. It follows from Lemma 2.5 that we may assume that $f^{\prime} \not \equiv 0$. It is then enough to prove that every infinite subset $E$ of $D$ has an element $a$ which satisfies the property given in Proposition 4.3. Assume that there exists a subset $E$ which does not have such an element $a$. Replacing $E$ by $E \backslash\left\{a \mid f^{\prime}(a)=0\right\}$, we have

$$
\forall a \in E \exists \mathfrak{m}_{1}, \ldots, \mathfrak{m}_{s} \forall \mathfrak{m} \in \operatorname{Max}(D) \backslash\left\{\mathfrak{m}_{1}, \ldots, \mathfrak{m}_{s}\right\} \forall x \in E \backslash\{a\} x-a \notin \mathfrak{m} .
$$

Fix two distinct elements $a$ and $b$ in $E$. It follows from the hypothesis on $E$ that there exists a finite subset $T$ of $\operatorname{Max}(D)$ such that for every $\mathfrak{m} \in \operatorname{Max}(D) \backslash T$ and every $x \in E \backslash\{a, b\}, x-a$ and $x-b$ do not belong to $\mathfrak{m}$. Since we are looking for a contradiction, we may replace $D$ with the ring $D_{T}$ and assume that

$$
\forall x \in E \backslash\{a, b\} \quad(x-a)(x-b) \in U(D),
$$

where $U(D)$ denotes the group of units of $D$ (in fact the group of $S \cup T$-units of $K)$.

For each $x \in E \backslash\{a, b\}$, let $X=\frac{x-a}{b-a}$ and $X^{*}=\frac{b-x}{b-a}$. Then $X, X^{*} \in U(D)$ and $X+X^{*}=1$. Furthermore, the hypothesis on $E$ implies that there are infinitely many such pairs. But we know (see for instance [7, Thm. 7.19]) that there are only finitely many pairs of separable non-constant units $\left(U, U^{*}\right)$ of $D$ such that $U+U^{*}=1$ and that all the solutions of $Y+Y^{*}=1$ in non-constant units are of the form $\left(U^{p^{m}}, U^{* p^{m}}\right)$ where $m \geq 0$. Consequently, there exists at least one pair $\left(U_{0}, U_{0}^{*}\right)$ of separable units such that $U_{0}+U_{0}^{*}=1$ and such that, for infinitely many $m \geq 0,\left(U_{0}^{p^{m}}, U_{0}^{* p^{m}}\right)$ is some of the previous pairs $\left(X, X^{*}\right)$. Thus we may consider a strictly increasing sequence of integers $\left\{m_{k}\right\}_{k \geq 0}$ such that

$$
U_{0}^{p^{m_{k}}}=X_{m_{k}}=\frac{x_{m_{k}}-a}{b-a} \text { with } x_{m_{k}} \in E .
$$

Let $q=p^{f}$ be the cardinality of the constant field of $K$. From the infinite sequence $\left\{m_{k}\right\}_{k \geq 0}$, we may extract another infinite strictly increasing sequence $\left\{t_{k}\right\}_{k \geq 0}$ such that $t_{k} \equiv t_{0}(\bmod f)$. Let us write $t_{k}=t_{0}+f r_{k}$. Then

$$
X_{t_{k}}=U_{0}^{p^{t_{k}}}=U_{0}^{p^{t_{0}} \times q^{r_{k}}}=X_{t_{0}}^{q^{r_{k}}} .
$$

We also know (see for instance [7, Thm. 5.12]) that there exists $l_{0}$ such that for every $l \geq l_{0}$, the function field $K$ has at least one maximal ideal $\mathfrak{m}_{l}$ with norm $q^{l}$. Finally, for $k$ large enough, we have

$$
X_{t_{k}}-X_{t_{0}}=X_{t_{0}}^{q^{r_{k}}}-X_{t_{0}} \in \mathfrak{m}_{r_{k}} .
$$

Equivalently, for $k$ large enough, $x_{t_{k}}-x_{t_{0}} \in \mathfrak{m}_{r_{k}}$. Thus, the element $x_{t_{0}} \in E$ leads to a contradiction to the assumption on $E$.

\section{ACKNOWLEDGMENT}

The authors thank David Adam for his advice about function fields.

\section{REFERENCES}

[1] Y. Fares, $\delta$-rings and factorial sequences preservation, Acta Arith., 123 (2006), 377-388. MR2262251 (2008a:13012)

[2] W. Feit, On symmetric balance incomplete block designs with doubly transitive automorphism groups, J. Combin. Theory Ser A, 14 (1973), 221-247. MR0327540 (48:5882)

[3] M. Fried, On Hilbert's irreducibility theorem, J. Number Theory, 6 (1974), 211-231. MR0349624 (50:2117) 
[4] K. Kubota, Note on a conjecture of W. Narkiewicz, J. Number Theory, 4 (1972), 181-190. MR0309903 (46:9007)

[5] P. Müller, Kronecker conjugacy of polynomials, Trans. Amer. Math. Soc., 350 (1998), 18231850. MR:1458331 (98h:11032)

[6] W. Narkiewicz, Polynomial mappings, Lecture Notes in Math., 1600, Springer-Verlag, Berlin, 1995. MR1367962 (97e:11037)

[7] M. Rosen, Number Theory in Function Fields, Springer-Verlag, New York, 2002. MR.1876657 (2003d:11171)

[8] J. F. Voloch, The equation $a x+b y=1$ in characteristic $p$, J. Number Theory, 73 (1998), 195-200. MR.1658019 (2000b:11029)

Département de Mathématiques, LAMFA CNRS-UMR 6140, Université de Picardie, 80039 Amiens, France

E-mail address: jean-luc.chabert@u-picardie.fr

Département de Mathématiques, LAMfa CNRS-UMR 6140, Université de Picardie, 80039 Amiens, France

E-mail address: youssef.fares@u-picardie.fr 